\title{
COHOMOLOGY OF MAXIMAL IDEAL SPACES
}

\author{
BY ANDREW BROWDER
}

\author{
Communicated by I. M. Singer, July 14, 1961
}

Let $A$ be a commutative Banach algebra with unit, and let $M$ be the maximal ideal space of $A$. We say that $A$ is generated by $x_{1}, \cdots, x_{n}$ if the polynomials $p\left(x_{1}, \cdots, x_{n}\right)$ form a dense subalgebra of $A$. Let $H^{i}(M, C)$ denote the $j$ th $\check{C}^{2}$ ch cohomology group of $M$ with complex coefficients.

THEOREM. If $A$ is generated by $n$ elements, then $H^{i}(M, C)=0$ for $j \geqq n$.

Proof. If $x_{1}, \cdots, x_{n}$ generate $A$, then the map of $M$ into $C^{n}$ given by $h \rightarrow\left(h\left(x_{1}\right), \cdots, h\left(x_{n}\right)\right)$ is a homeomorphism of $M$ onto a compact set $K$. It is known (see, e.g., [1]) that $K$ is polynomially convex, i.e., if $V$ is any open set containing $K$, there exists an analytic polyhedron $U$ defined by polynomials, such that $K \subset U \subset V$. Each such polyhedron $U$ is a domain of holomorphy (Stein manifold) and a Runge domain. For any $n$-dimensional Stein manifold $U$, it is known that $H^{j}(U, C)=0$ for $j>n$. (See [2] for a proof.) For any Runge domain $U$ in $C^{n}$, Serre has shown [3] that $H^{n}(U, C)=0$. The proof is completed by observing the following nonstandard but elementary continuity property of Cech cohomology:

FACT. Let $X$ be a compact subset of a metric space, $G$ an abelian group, $j$ a non-negative integer. If for every open set $V \supset K$, there exists an open $U$ with $K \subset U \subset V$ and $H^{i}(U, G)=0$, then $H^{i}(K, G)=0$.

Corollary. Let $M$ be an n-dimensional compact orientable manifold. Let $C(M)$ denote the ring of all continuous complex-valued functions on $M$, normed by the sup norm. Then $C(M)$ requires at least $n+1$ generators.

REMARKs. 1. For $n=1$, the condition of the theorem is both necessary and sufficient; a compact subset $K$ of the plane is polynomially convex if and only if $K$ has connected complement, which is equivalent to $H^{1}(K, C)=0$.

2. It is of course trivial that at least $n+1$ real-valued functions are required to generate $C(M)$ when $M$ is a compact $n$-dimensional manifold, but it should be observed that in general, a compact space $X$ need not require as many complex functions to generate $C(X)$ as it does real functions. Example: If $X$ is a compact connected plane set 
with no interior and connected complement, $C(X)$ is generated by the single function $z$ (Mergelyan's theorem); but $C(X)$ is generated by a single real function if and only if $X$ is a Jordan arc.

3. The author is unaware of any other proof of the corollary even for the case $M$ the two-sphere.

\section{REFERENCES}

1. R. Arens and A. P. Calderón, Analytic functions of several Banach algebra elements, Ann. of Math. vol. 62 (1955) pp. 204-216.

2. Séminaires de H. Cartan, 1951-1952.

3. J.-P. Serre, Une propriêté topologique des domaines de Runge, Proc. Amer. Math. Soc. vol. 6 (1955) pp. 133-134.

BROWN UNIVERSITY

ENGINEER
or
MATHEMATICIAN
The Radiation Laboratory
of the Johns Hopkins University
has a Position for an
Engineer or
Applied Mathematician
experience in the field of:
SIGNAL DETECTION
AND ANALYSIS
With Emphasis on Sequential
Detection and Characterization
of Complex Signals
Favorable Arrangements for
Advanced Study in the
University Graduate Schools.
Excellent Laboratory Facilities
Broad Opportunity for
Career Development
Address Inquiries To:
RADIATION LABORATORY
THE JOHNS HOPKINS
UNIVERSITY
Homewood Campus
Baltimore 18, Md.

\section{ILLINOIS JOURNAL OF MATHEMATICS}

A quarterly journal of basic research in pure and applied mathematics published by the University of Illinois, Urbana.

\section{edited by}

REINHOLD BAER • PAUL T. BATEMAN

S. S. CHERN • J. L. DOOB

A. H. TAUB • GEORge W. WHITEHEAD

The subscription price is $\$ 9.00$ a volume (four numbers); this is reduced to $\$ 5.00$ for individual members of the American Mathematical Society. Subscriptions should be sent to the University of Illinois Press, Urbana, Illinois. 